\title{
Effects of Protocol Design on Lactate Minimum Power
}

\author{
Authors \\ M. A. Johnson, G. R. Sharpe \\ Affiliation \\ Nottingham Trent University, School of Science and Technology, Nottingham, United Kingdom
}

Key words

- lactate minimum

- test protocol

- cycling accepted after revision October 28, 2010

Bibliography DOI http://dx.doi.org/ 10.1055/s-0030-1268487 Published online: December 16, 2010 Int J Sports Med 2011; 32: 199-204 @ Georg Thieme Verlag KG Stuttgart · New York ISSN 0172-4622

\section{Correspondence}

\section{Dr. Michael Alan Johnson} Nottingham Trent University School of Science and Technology Clifton Campus

NG11 8NS Nottingham United Kingdom Tel.: + 44/11/5848 3362 Fax: +44/11/5848 6636 michael.johnson@ntu.ac.uk

\section{Abstract \\ $\nabla$}

The aim of this investigation was to use a validated lactate minimum test protocol and evaluate whether blood lactate responses and the lactate minimum power are influenced by the starting power (study 1 ) and $1 \mathrm{~min}$ inter-stage rest intervals (study 2) during the incremental phase. Study 1: 8 subjects performed a lactate minimum test comprising a lactate elevation phase, recovery phase, and incremental phase comprising 5 continuous 4 min stages with starting power being $40 \%$ or $45 \%$ of the maximum power achieved during the lactate elevation phase, and with power increments of $5 \%$ maximum power.

\section{Introduction}

\section{$\nabla$}

The maximal lactate steady state (MLSS) represents the highest exercise intensity at which blood lactate concentration $\left(\left[\mathrm{lac}^{-}\right]_{\mathrm{B}}\right)$ remains stable over time and is widely acknowledged as an important determinant of endurance exercise performance [11-13]. Unfortunately, direct MLSS determination is laborious, requiring multiple, constant-intensity exercise tests (typically lasting $30 \mathrm{~min}$ ) over a range of exercise intensities. Numerous protocols have therefore been designed to predict MLSS using a single exercise test $[11,25]$. The lactate minimum test was originally proposed by Tegtbur et al. [26] as a method to predict MLSS running velocity, and the protocol has since been adapted for MLSS prediction in cycling [11,13,21,22,24] and swimming [15]. The lactate minimum represents the workload corresponding to the nadir of a U-shaped $\left[\mathrm{lac}^{-}\right]_{\mathrm{B}}$ profile during an incremental exercise test (hereafter termed incremental phase) initiated with hyperlactataemia due to a preceding high-intensity exercise bout (hereafter termed lactate elevation phase) and short recovery (hereafter
Study 2: 8 subjects performed 2 identical lactate minimum tests except that during one of the tests the incremental phase included $1 \mathrm{~min}$ inter-stage rest intervals. The lactate minimum power was lower when the incremental phase commenced at $40 \%(175 \pm 29 \mathrm{~W})$ compared to $45 \%(184 \pm 30 \mathrm{~W})$ maximum power $(\mathrm{p}<0.01)$, and was increased when $1 \mathrm{~min}$ inter-stage rest intervals were included during the incremental phase ( $192 \pm 25$ vs. $200 \pm 26 \mathrm{~W}, \mathrm{p}<0.01)$. In conclusion, changes in lactate minimum power were small and thus unlikely to compromise test validity and therefore training status evaluation and exercise prescription. 
Whether the inclusion of rest intervals during the incremental phase affects the lactate minimum workload remains unknown. The intensity at which the incremental phase commences has also varied and Carter et al. [4] concluded that starting intensity affected lactate minimum running speed. However, during the incremental phase $\left[\mathrm{lac}^{-}\right]_{\mathrm{B}}$ reached, or approached, that seen at rest, which prevents a genuine lactate minimum nadir being observed and therefore renders that study inconclusive [13].

Therefore, using our recently described, validated lactate minimum test protocol [11], the aims of this investigation were to examine whether $\left[\mathrm{lac}^{-}\right]_{\mathrm{B}}$ responses and the lactate minimum power are affected by using different incremental phase starting powers (study 1 ) and the introduction of $1 \mathrm{~min}$ inter-stage rest intervals during the incremental phase (study 2 ).

\section{Method}

$\nabla$

\section{Participants, equipment and measurements}

Studies 1 and 2 were approved by the Nottingham Trent University ethics committee, and were conducted in accordance with the principles of the Declaration of Helsinki and the International Journal of Sports Medicine [8]. 16 non-smoking recreationally active (performing 30-90 min of aerobic exercise, 3-4 times per week) male subjects provided written informed consent to participate in the study. Subjects refrained from strenuous exercise during the $24 \mathrm{~h}$ preceding an exercise test. On test days subjects abstained from alcohol and caffeine and reported to the laboratory at least $2 \mathrm{~h}$ post-prandial. Successive tests were separated by at least $48 \mathrm{~h}$, but no more than 1 week, and were performed at a similar time of day. Tests within each study were performed in a counterbalanced order.

Exercise was performed on an electromagnetically-braked cycle ergometer (Excalibur Sport, Lode, Groningen, The Netherlands). The same self-selected cycling cadence $\left(83 \pm 8 \mathrm{revs} \cdot \mathrm{min}^{-1}\right)$ was used during all tests. Arterialised venous blood samples were taken from a dorsal hand vein via an indwelling cannula and analysed for $\left[\mathrm{lac}^{-}\right]_{\mathrm{B}}$ (P-GM7 MicroStat, Analox Instruments, London, UK). During the lactate elevation phase, subjects wore a facemask (model 7940, Hans Rudolph, Kansas City, Missouri) and respiratory variables were measured breath-by-breath (Pulmolab EX670, Ferraris Respiratory Europe, Hertford, UK).

Study 1 - effect of different starting powers during the incremental phase

Subjects ( $\mathrm{n}=8$; age 23.6. \pm 4.5 years; height $179.4 \pm 4.4 \mathrm{~cm}$; body mass $79.6 \pm 8.1 \mathrm{~kg}$ ) performed 2 lactate minimum tests as described in Johnson et al. [11]. Each test comprised 3 consecutive phases: (I) lactate elevation phase comprising maximal incremental exercise; (II) 8 min recovery phase at $60 \mathrm{~W}$; and (III) incremental phase comprising five 4 min exercise stages, starting at either $40 \%$ or $45 \%$ of the maximal power ( $\dot{W}$ max) achieved during the lactate elevation phase, and with power increments of $5 \% \dot{W} \max$ (hereafter these tests are termed $\mathrm{LM}_{40-60}$ and $\mathrm{LM}_{45-65}$, respectively). During the lactate elevation phase power was increased every $15 \mathrm{~s}$ by a constant amount ( $8-10 \mathrm{~W}$, depending upon the subjects training history) chosen to elicit exercise intolerance (cadence $<60 \mathrm{revs} \cdot \mathrm{min}^{-1}$ ) in $\sim 10 \mathrm{~min}$. The final power and the highest oxygen uptake recorded over any $30 \mathrm{~s}$ period defined Ẇmax and maximal oxygen uptake, respectively. During their second lactate minimum test, subjects were encouraged to exercise for the same duration during the lactate eleva- tion phase as that achieved in the initial lactate minimum test, at which point the lactate elevation phase was terminated. Exercise duration during the lactate elevation phase was matched in an attempt to replicate the magnitude of lactate elevation, and thus the starting $\left[\mathrm{lac}^{-}\right]_{\mathrm{B}}$ at the onset of the incremental phase. We did not conduct a lactate minimum test in which the incremental phase began at $50 \% \dot{W}$ max because pilot work showed that $70 \% \dot{W} \max$ (which would represent the final stage of the incremental phase) represented an intolerable workload. Blood samples were taken every minute following the first minute of the recovery phase.

\section{Study 2 - effect of inter-stage rest interval during the incremental phase}

Subjects $(n=8$; age 24.5. \pm 5.8 years; height $177.3 \pm 6.0 \mathrm{~cm}$; body mass $77.5 \pm 7.0 \mathrm{~kg}$ ) performed 2 lactate minimum tests as described for $\mathrm{LM}_{45-65}$. The 2 tests were performed with $\left(\mathrm{LM}_{\mathrm{INT}}\right)$ and without $\left(\mathrm{LM}_{\mathrm{CONT}}\right) 1 \mathrm{~min}$ inter-stage rest intervals during the incremental phase. During their second lactate minimum test, subjects were again encouraged to exercise for the same duration during the lactate elevation phase as that achieved in the initial lactate minimum test. Blood samples were taken every minute during the incremental phase.

\section{Data analyses}

Data analyses were performed using SPSS. The Shapiro-Wilk test was used to confirm that the data were normally distributed. The area under the $\left[\mathrm{lac}^{-}\right]_{\mathrm{B}}$ curve in the recovery phase of study 1 was calculated using the trapezoidal rule. The lactate minimum power was determined from the zero gradient tangent to a cubic spline function fitting the $\left[\mathrm{lac}^{-}\right]_{\mathrm{B}}$ (measured at the end of each stage) vs. power data. Rates of change of $\left[\operatorname{lac}^{-}\right]_{B}\left(\Delta\left[\operatorname{lac}^{-}\right]_{B} / \Delta t\right.$, where $\mathrm{t}=$ time) during each incremental phase stage were taken as the gradient of a linear regression of $\left[\mathrm{lac}^{-}\right]_{\mathrm{B}}$ against time. Data were analysed using repeated measures ANOVA and paired ttests where appropriate. Pearson product-moment correlation coefficients ( $r$ ) were determined to assess the relationship between variables. Results are reported as mean \pm SD. Statistical significance was set at $\mathrm{p}<0.05$.

\section{Results}

$\nabla$

The $\left[\operatorname{lac}^{-}\right]_{\mathrm{B}}$ profile during the incremental phase was well described by the cubic spline function (study $1: R^{2}=0.96 \pm 0.04$ and $0.97 \pm 0.04$ in $\mathrm{LM}_{40-60}$ and $\mathrm{LM}_{45-65}$, respectively; study 2: $\mathrm{R}^{2}=0.99 \pm 0.02$ and $0.92 \pm 0.07$ in $\mathrm{LM}_{\mathrm{CONT}}$ and $\mathrm{LM}_{\mathrm{INT}}$, respectively), with $\mathrm{R}^{2}$ values in $\mathrm{LM}_{\text {CONT }}$ being higher than those in $\mathrm{LM}_{\mathrm{INT}}$ $(\mathrm{p}<0.05)$. Lactate minimum power was correlated $(\mathrm{p}<0.01)$ with $\dot{W} \max \left(r=0.99\right.$ in both $\mathrm{LM}_{40-60}$ and $\mathrm{LM}_{45-65}$, and $\mathrm{r}=0.97$ and 0.99 in $\mathrm{LM}_{\mathrm{CONT}}$ and $\mathrm{LM}_{\mathrm{INT}}$, respectively).

\section{Study 1}

The $\dot{W} \max$ and maximal oxygen uptake during the first lactate minimum test were $344 \pm 56 \mathrm{~W}$ and $3.67 \pm 0.45 \mathrm{~L} \cdot \mathrm{min}^{-1}$, respectively. For each subject exercise duration during the lactate elevation phase was identical in both lactate minimum tests. All subjects demonstrated a declining $\left[\operatorname{lac}^{-}\right]_{B}$ during the recovery phase ( $\bullet$ Fig. 1) and the calculated area under the $\left[\mathrm{lac}^{-}\right]_{\mathrm{B}}$ curve in $\mathrm{LM}_{40-60}(46 \pm 6 \mathrm{mmol})$ was not different from that determined in $\mathrm{LM}_{45-65}(47 \pm 7 \mathrm{mmol})$. The $\left[\mathrm{lac}^{-}\right]_{\mathrm{B}}$ at the end of the recovery phase was $6.8 \pm 1.1 \mathrm{mmol} \cdot \mathrm{L}^{-1}$ (range: $5.2-8.2 \mathrm{mmol} \cdot \mathrm{L}^{-1}$ ) in 

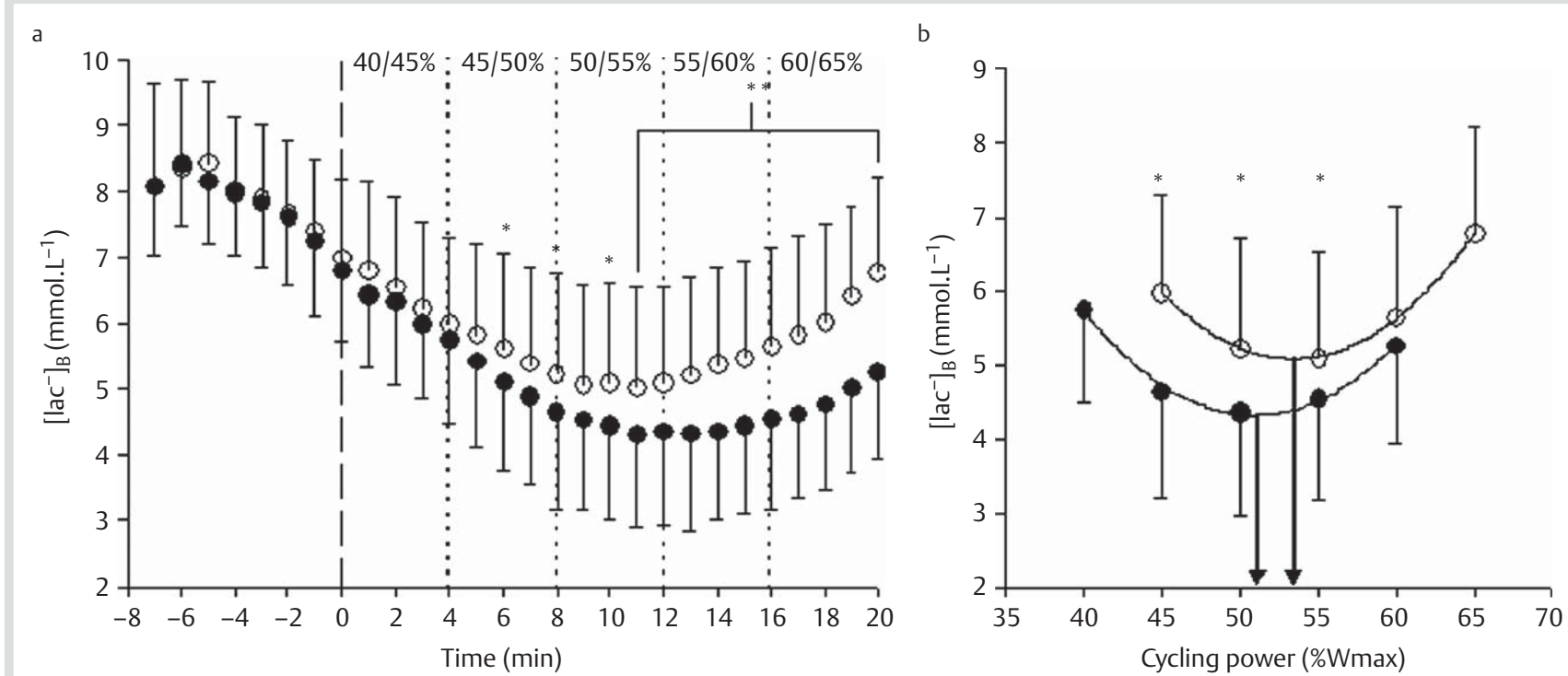

Fig. 1 a Blood lactate concentration $\left(\left[\operatorname{lac}^{-}\right]_{B}\right)$ throughout the recovery and incremental phases of $\mathrm{LM}_{40-60}(\bullet)$ and $\mathrm{LM}_{45-65}(\mathrm{o})$. Dashed vertical line at 0 min denotes transition from recovery to incremental phase. All other dashed lines denote the transition between consecutive exercise stages. Relative cycling powers (\% $\dot{W}$ max ) are shown above each 4 min epoch. $\mathbf{b}$ The effect of different starting powers on the U-shaped $\left[\text { lac }^{-}\right]_{B}$ profile and the lactate minimum (denoted by arrows) during $\mathrm{LM}_{40-60}(\bullet)$ and $\mathrm{LM}_{45-65}(\mathrm{o})$. Values are mean $\pm \mathrm{SD}$. Difference between trials, ${ }^{*} \mathrm{p}<0.05,{ }^{* *} \mathrm{p}<0.01$.

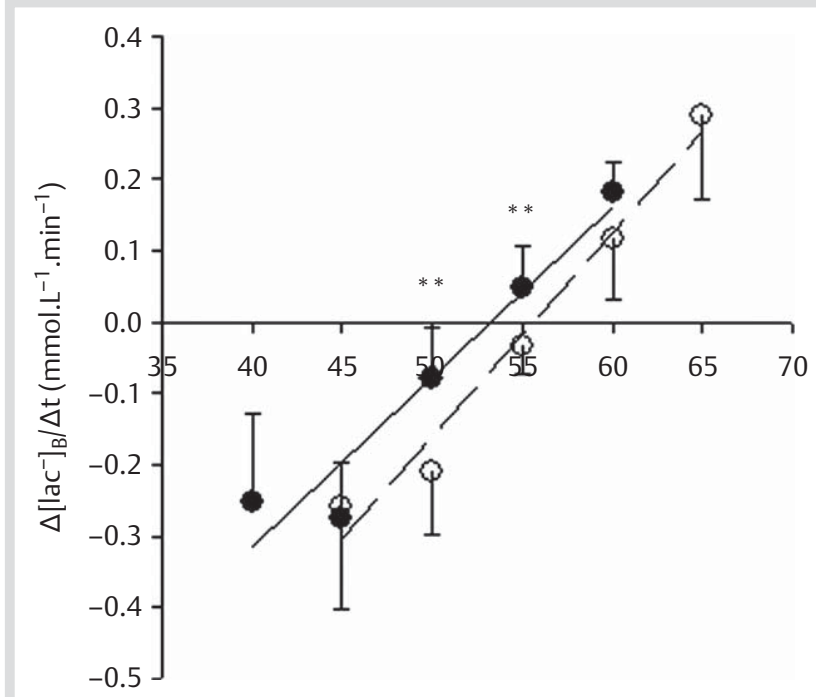

Cycling power (\%Wmax)

Fig. 2 Rates of change of blood lactate concentration $\left(\Delta\left[\operatorname{lac}^{-}\right]_{B} / \Delta t\right)$ during each incremental phase stage of $\mathrm{LM}_{40-60}(\bullet)$ and $\mathrm{LM}_{45-65}$ (o). Values are mean \pm SD. Difference between trials, ${ }^{* *} \mathrm{p}<0.01$.

$\mathrm{LM}_{40-60}$ and $7.0 \pm 1.2 \mathrm{mmol} \cdot \mathrm{L}^{-1}$ (range: $5.2-8.7 \mathrm{mmol} \cdot \mathrm{L}^{-1}$ ) in $\mathrm{LM}_{45-65}$. The lactate minimum power was lower in $\mathrm{LM}_{40-60}$ $(175 \pm 29 \mathrm{~W}, 50.9 \pm 0.9 \% \mathrm{~W} \max )$ compared to $\mathrm{LM}_{45-65}(184 \pm 30 \mathrm{~W}$, $53.5 \pm 1.2 \%$ Wmax $)(\mathrm{p}<0.01)$ ( $\bullet$ Fig. 1). The lactate minimum $\left[\mathrm{lac}^{-}\right]_{\mathrm{B}}$ was lower in $\mathrm{LM}_{40-60}\left(4.3 \pm 1.4 \mathrm{mmol} \cdot \mathrm{L}^{-1}\right.$, range: $3.0-$ $\left.6.9 \mathrm{mmol} \cdot \mathrm{L}^{-1}\right)$ compared to $\mathrm{LM}_{45-65}\left(5.1 \pm 1.5 \mathrm{mmol} \cdot \mathrm{L}^{-1}\right.$, range $\left.3.8-8.4 \mathrm{mmol} \cdot \mathrm{L}^{-1}\right)(\mathrm{p}<0.01)$. The time taken to reach the lactate minimum from the start of the incremental phase was longer in $\mathrm{LM}_{40-60}(12.74 \pm 0.77 \mathrm{~min})$ compared to $\mathrm{LM}_{45-65}(10.65 \pm 0.91 \mathrm{~min})$ $(\mathrm{p}<0.01) . \Delta\left[\mathrm{lac}^{-}\right]_{\mathrm{B}} / \Delta \mathrm{t}$ was higher in $\mathrm{LM}_{40-60}$ compared to $\mathrm{LM}_{45-66}$ at $50 \%$ and $55 \% \dot{\mathrm{W} m a x}(\mathrm{p}<0.01)$ ( $\bullet$ Fig. 2 ).

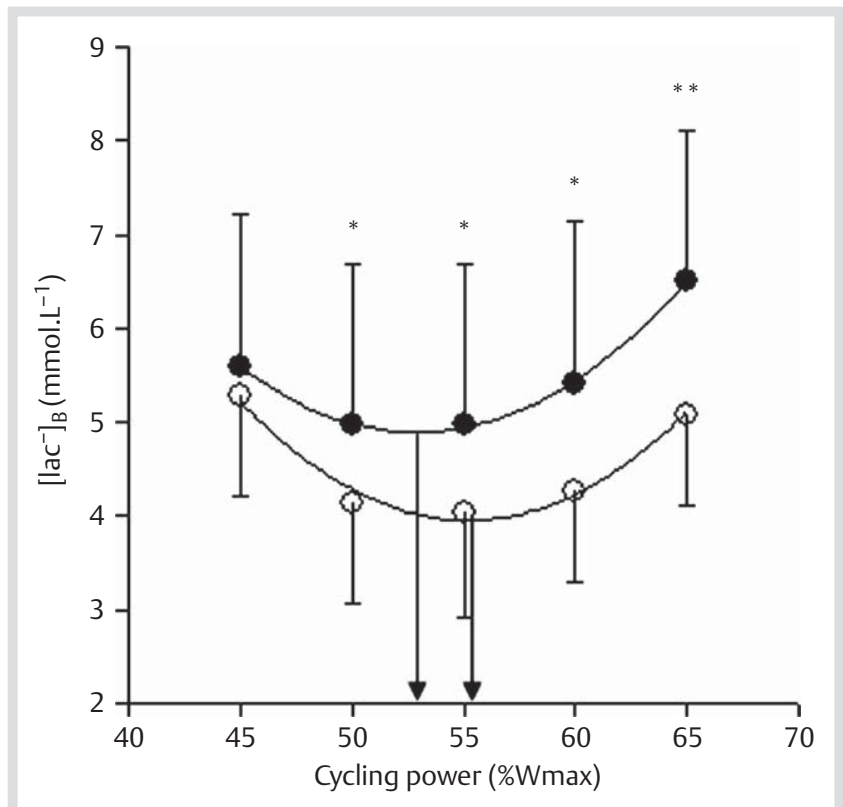

Fig. 3 The effect of inter-stage rest intervals during the incremental phase on the U-shaped blood lactate concentration $\left(\left[\mathrm{lac}^{-}\right]_{\mathrm{B}}\right)$ profile and the lactate minimum (denoted by arrows). $\bullet, \mathrm{LM}_{\mathrm{CONT}}$; o, $\mathrm{LM}_{\mathrm{INT}}$. Values are mean \pm SD. Difference between trials, ${ }^{*} p<0.05,{ }^{* *} p<0.01$.

\section{Study 2}

The $\dot{W} \max$ and maximal oxygen uptake during the first lactate minimum test were $363 \pm 49 \mathrm{~W}$ and $3.64 \pm 0.49 \mathrm{~L} \cdot \mathrm{min}^{-1}$, respectively. Exercise duration during the lactate elevation phase was identical in both lactate minimum tests. The $\left[\mathrm{lac}^{-}\right]_{\mathrm{B}}$ at the end of the recovery phase was $6.7 \pm 1.6 \mathrm{mmol} \cdot \mathrm{L}^{-1}$ (range: $4.6-$ $9.8 \mathrm{mmol} \cdot \mathrm{L}^{-1}$ ) in $\mathrm{LM}_{\mathrm{CONT}}$ and $6.4 \pm 1.1 \mathrm{mmol} \cdot \mathrm{L}^{-1}$ (range: 4.9 $8.3 \mathrm{mmol} \cdot \mathrm{L}^{-1}$ ) in $\mathrm{LM}_{\mathrm{INT}}$. The lactate minimum power was higher in $\mathrm{LM}_{\mathrm{INT}}(200 \pm 26 \mathrm{~W}, 55.3 \pm 1.1 \%$ Wmax $)$ compared to $\mathrm{LM}_{\mathrm{CONT}}$ $(192 \pm 25 \mathrm{~W}, 52.9 \pm 1.9 \% \dot{\mathrm{W}} \max )(\mathrm{p}<0.01)$ (๑ Fig. 3). The lactate minimum $\left[\mathrm{lac}^{-}\right]_{\mathrm{B}}$ was lower in $\mathrm{LM}_{\mathrm{INT}}\left(4.0 \pm 1.1 \mathrm{mmol} \cdot \mathrm{L}^{-1}\right.$, range: 
2.5-6.0 $\left.\mathrm{mmol} \cdot \mathrm{L}^{-1}\right)$ compared to $\mathrm{LM}_{\mathrm{CONT}}\left(4.9 \pm 0.7 \mathrm{mmol} \cdot \mathrm{L}^{-1}\right.$, range: $\left.2.9-8.6 \mathrm{mmol} \cdot \mathrm{L}^{-1}\right)(\mathrm{p}<0.05)$. Following the first stage of the incremental phase, $\left[\mathrm{lac}^{-}\right]_{\mathrm{B}}$ remained lower in $\mathrm{LM}_{\mathrm{INT}}$ compared to $\operatorname{LM}_{\mathrm{CONT}}$ ( 0 Fig. 4). Furthermore, during the latter 3 stages of the incremental phase in $\mathrm{LM}_{\mathrm{INT}}$ an increasing oscillatory response in $\left[\mathrm{lac}^{-}\right]_{\mathrm{B}}$ was observed. Thus $\Delta\left[\mathrm{lac}^{-}\right]_{\mathrm{B}} / \Delta \mathrm{t}$ was determined only over 3-5 min of each incremental phase stage. Differences in $\Delta\left[\mathrm{lac}^{-}\right]_{\mathrm{B}} / \Delta \mathrm{t}$ between $\mathrm{LM}_{\mathrm{CONT}}$ and $\mathrm{LM}_{\mathrm{INT}}$ increased as the incremental phase progressed ( $\bullet$ Fig. 5 ), and a significant main effect for trial was observed with $\Delta\left[\mathrm{lac}^{-}\right]_{\mathrm{B}} / \Delta \mathrm{t}$ during $\mathrm{LM}_{\mathrm{INT}}$ being greater than that measured in $\mathrm{LM}_{\mathrm{CONT}}(\mathrm{p}<0.05)$.

\section{Discussion}

$\nabla$

The main finding of this investigation was that despite $\left[\operatorname{lac}^{-}\right]_{B}$ responses being modified by starting the incremental phase at a lower power (study 1 ) and including $1 \mathrm{~min}$ inter-stage rest intervals during the incremental phase (study 2 ), the effects on lactate minimum power are small.

The $9 \mathrm{~W}$ reduction in lactate minimum power when the incremental phase was initiated at a lower intensity is consistent with

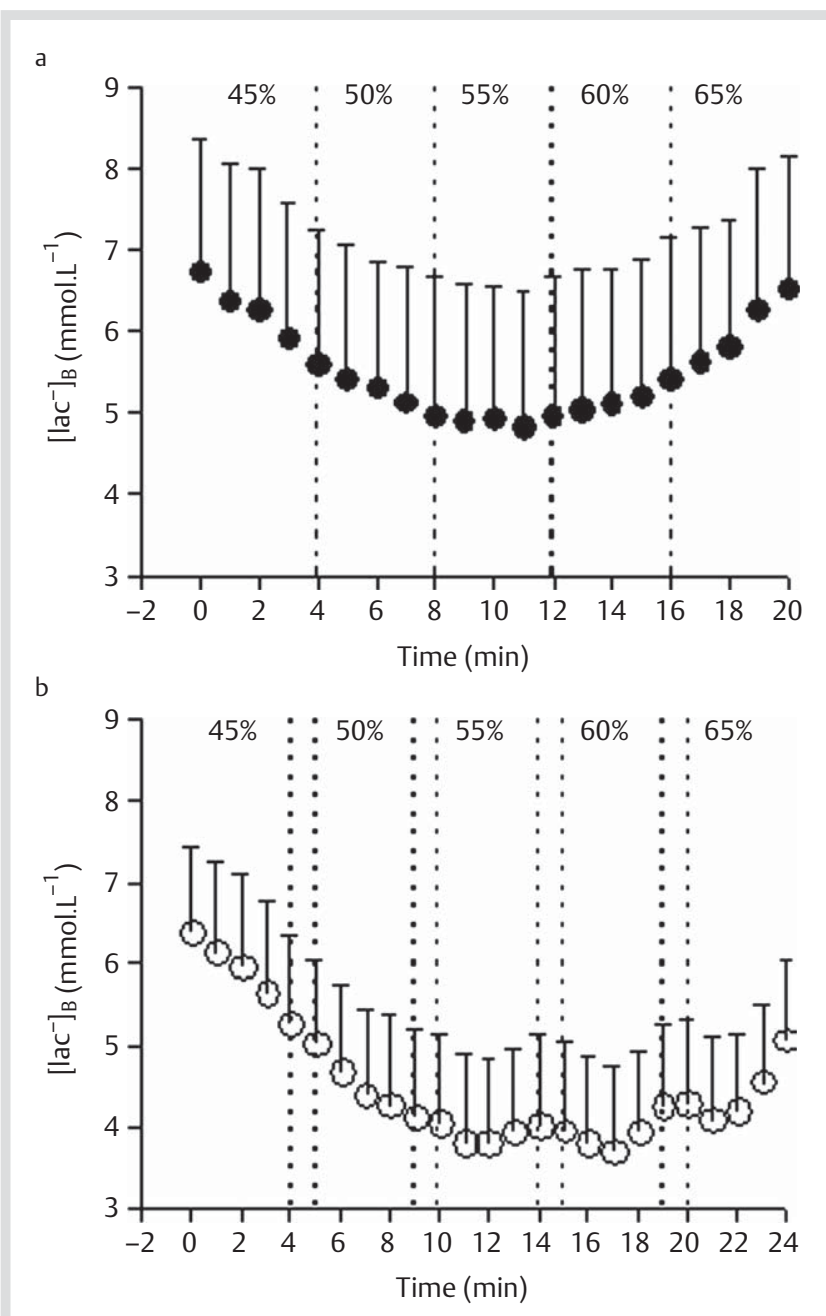

Fig. 4 Blood lactate concentration ([lac $\left.\left.{ }^{-}\right]_{B}\right)$ throughout the incremental phase of $\operatorname{LM}_{\text {CONT }}(\mathbf{a})$ and $\mathrm{LM}_{\mathrm{INT}}(\mathbf{b})$. Dashed vertical lines in panel A denote the transition between consecutive exercise stages. Dashed vertical lines in panel B denote 1 min rest intervals. Relative cycling powers (\% Ẃmax) are shown above each 4 min epoch. Values are mean \pm SD. the findings of Carter et al. [4], although in this study $\left[\operatorname{lac}^{-}\right]_{\mathrm{B}}$ reached, or approached, resting values during the incremental phase, which according to MacIntosh et al. [13] renders their study inconclusive. Conversely, in study $1\left[\mathrm{lac}^{-}\right]_{\mathrm{B}}$ remained $\geq 3.0 \mathrm{mmol} \cdot \mathrm{L}^{-1}$ in all subjects throughout the incremental phase, thus indicating a genuine influence of starting intensity on the lactate minimum power. That our protocol [11] standardises starting intensity for the incremental phase is therefore advantageous in comparison to protocols in which knowledge of training status $[1,12,26]$ or separate testing $[5,13,15,20,22]$ was required. However, the difference in lactate minimum power between $\mathrm{LM}_{40-60}$ and $\mathrm{LM}_{45-65}$ is well within the $95 \%$ limits of agreement (22W) for the comparison of lactate minimum (from $\mathrm{LM}_{45-65}$ ) and MLSS powers [11] and is also less than the resolution with which MLSS is typically determined (10-20W) [25]. Thus using $\mathrm{LM}_{40-60}$ is unlikely to compromise test validity, and therefore training status evaluation or exercise prescription. On the contrary, given that $\mathrm{LM}_{45-65}$ could be considered physically challenging to complete, the lower intensities performed during the incremental phase of $\mathrm{LM}_{40-60}$ may provide a more tolerable test for certain individuals (e.g. untrained, elderly and/or clinical patients) $[14,18]$.

The greater decline in $\left[\mathrm{lac}^{-}\right]_{\mathrm{B}}$ when the incremental phase commenced at a lower power was probably due to greater lactate clearance by oxidative muscle $[2,4]$ since low intensity exercise accelerates lactate clearance $[2,16]$. Although the $\left[\mathrm{lac}^{-}\right]_{\mathrm{B}}$ was lower throughout the incremental phase of $\mathrm{LM}_{40-60}$ compared to $\mathrm{LM}_{45-65}, \Delta\left[\mathrm{lac}^{-}\right]_{\mathrm{B}} / \Delta \mathrm{t}$ was higher in $\mathrm{LM}_{40-60}$ at equivalent intensities (50\% and $55 \% \dot{\mathrm{W} m a x}$ ). This supports the notion that $\Delta\left[\mathrm{lac}^{-}\right]_{\mathrm{B}} /$ $\Delta t$ during each incremental phase stage does not solely reflect the metabolic demand of that exercise intensity, i.e., $\Delta\left[\mathrm{lac}^{-}\right]_{\mathrm{B}} / \Delta \mathrm{t}$ during constant power exercise [11]. Rather, $\Delta\left[\mathrm{lac}^{-}\right]_{\mathrm{B}} / \Delta \mathrm{t}$ is likely to be influenced by the underlying blood lactate recovery kinetics following the lactate elevation phase and preceding exercise stage $[4,11]$. Therefore, at a given relative power, the greater exercise history during $\mathrm{LM}_{40-60}$ compared to $\mathrm{LM}_{45-65}$ may have promoted greater lactate release from passive lactate "reservoirs" (i.e., resting skeletal muscle) and active muscle [6], thus causing a slight leftward shift of the lactate minimum curve.

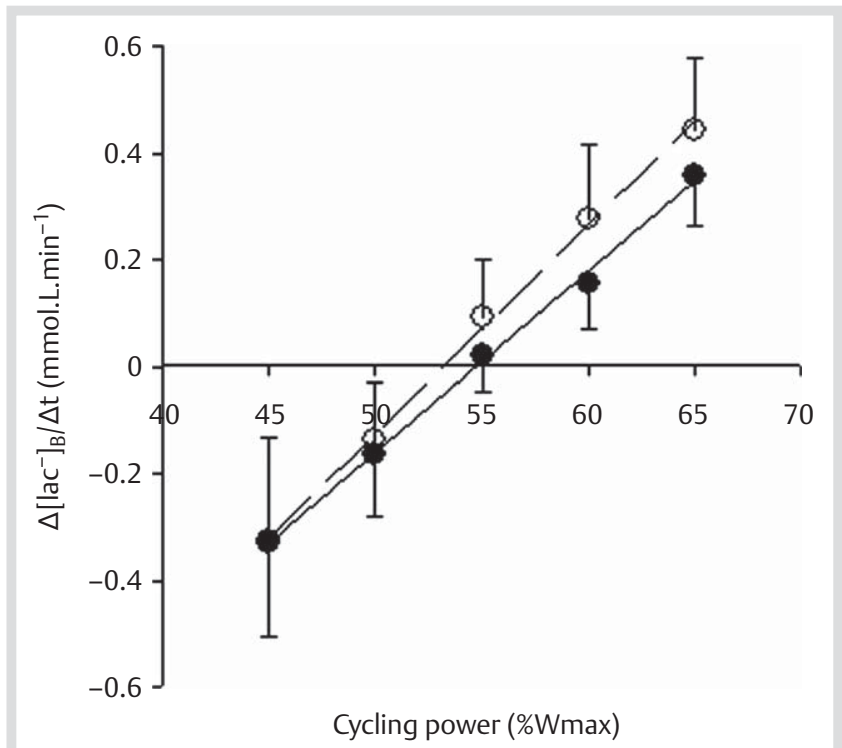

Fig. 5 Rates of change of blood lactate concentration $\left(\Delta\left[\operatorname{lac}^{-}\right]_{B} / \Delta t\right)$ during each incremental phase stage of $\mathrm{LM}_{\mathrm{CONT}}(\bullet)$ and $\mathrm{LM}_{\mathrm{INT}}(\mathrm{o}) \cdot \Delta\left[\mathrm{lac}^{-}\right]_{\mathrm{B}} / \Delta \mathrm{t}$ was determined over $3-5$ min of each stage. Values are mean \pm SD. 
The purpose of the recovery phase is to allow $\left[\operatorname{lac}^{-}\right]_{B}$ to decrease prior to the incremental phase. A $\left[\mathrm{lac}^{-}\right]_{\mathrm{B}}$ of $\sim 8.0 \mathrm{mmol} \cdot \mathrm{L}^{-1}$ at the onset of the incremental phase has been recommended [4], although a rationale was not provided. Although some subjects in our studies did not meet this criterion, a range of starting $\left[\mathrm{lac}^{-}\right]_{\mathrm{B}}$ values evidently permit a valid test $[11,13]$. It is, however, essential that the starting $\left[\mathrm{lac}^{-}\right]_{\mathrm{B}}$ is sufficiently high to prevent $\left[\mathrm{lac}^{-}\right]_{\mathrm{B}}$ approaching/reaching resting values during the incremental phase, which prevents the determination of a lactate minimum nadir.

Many studies have included inter-stage rest intervals during the incremental phase of either $30 \mathrm{~s}[16,26], 45 \mathrm{~s}[15,19]$, or more commonly $1 \mathrm{~min}[5,10,13,17,20,23]$. The $8 \mathrm{~W}$ increase in lactate minimum power with $1 \mathrm{~min}$ inter-stage rest intervals concurs with a previous study in which MLSS cycling power increased from $278 \mathrm{~W}$ to 300 and $310 \mathrm{~W}$ when rest intervals of 0.5 and $1.5 \mathrm{~min}$, respectively, were included every $5 \mathrm{~min}$ during each 30 min constant power MLSS determination test [3]. During incremental running exercise the speed at the $4.0 \mathrm{mmol} \cdot \mathrm{L}^{-1}$ $\left[\mathrm{lac}^{-}\right]_{\mathrm{B}}$ threshold was unchanged with $0.5 \mathrm{~min}$ inter-stage rest intervals [7], but increased by $\sim 0.70 \mathrm{~km} \cdot \mathrm{h}^{-1}$ with $1.5 \mathrm{~min}$ intervals [9]. Although increases in lactate transitions with test interruptions could result in an overestimate of endurance training status and an inappropriate prescription of training exercise intensity, the difference in lactate minimum power between $\mathrm{LM}_{\mathrm{CONT}}$ and $\mathrm{LM}_{\mathrm{INT}}$ is probably too small to have any practical significance (see above). Indeed, for reasons highlighted previously, the $\mathrm{LM}_{\mathrm{INT}}$ protocol would probably be more tolerable for certain individuals.

The lower $\left[\mathrm{lac}^{-}\right]_{\mathrm{B}}$ during $\mathrm{LM}_{\mathrm{INT}}$ compared to $\mathrm{LM}_{\mathrm{CONT}}$ probably resulted from a reduced glycolytic rate during the rest interval combined with a sustained metabolic rate promoting wholebody lactate clearance $[3,6] .4 \mathrm{~min}$ may have thus provided insufficient time for $\left[\mathrm{lac}^{-}\right]_{\mathrm{B}}$ to increase to the same value as that observed during $\mathrm{LM}_{\mathrm{CONT}}$, therefore causing a rightward shift of the lactate minimum curve. An increasing oscillatory response in $\left[\mathrm{lac}^{-}\right]_{\mathrm{B}}$ was observed in the latter incremental phase stages of $\mathrm{LM}_{\mathrm{INT}}$, and $\Delta\left[\mathrm{lac}^{-}\right]_{\mathrm{B}} / \Delta \mathrm{t}$ (determined over 3-5 min of each stage) was higher compared to $\mathrm{LM}_{\mathrm{CONT}}$ (differences in $\Delta\left[\mathrm{lac}^{-}\right]_{\mathrm{B}} / \Delta \mathrm{t}$ also approached statistical significance at $55 \%(\mathrm{p}=0.06)$ and $60 \%$ $(p=0.09) \dot{W}$ max $)$. A rapid decline in metabolic rate during each 1 min rest interval would have predisposed a delayed kinetic response (i.e., metabolic inertia) at the onset of the subsequent exercise stage $[18,27]$ and therefore, compared to $\mathrm{LM}_{\text {CONT, a }}$ larger contribution from non-oxidative energy pathways and thus greater lactate efflux from muscle.

Previous studies of lactate minimum test validity may have been influenced by the inclusion of rest intervals during the incremental phase and/or each 30 min constant load MLSS determination test. For example, MacIntosh et al. [13] included 1 min rest intervals during the incremental phase but not during MLSS determination tests. Although close agreement was observed between lactate minimum and MLSS powers, it is likely that lactate minimum power would have been lower had a continuous incremental phase been used (present study), whereas MLSS power would have been higher had $1 \mathrm{~min}$ rest intervals also accompanied each $5 \mathrm{~min}$ blood sample [3]. Furthermore, because changes in systemic lactate kinetics due to rest intervals are likely to differ between incremental exercise commencing with hyperlactataemia and $30 \mathrm{~min}$ square-wave constant load exercise [11], even identical rest interval durations may cause dissimilar shifts in lactate minimum and MLSS workloads [3,7], which may partly explain some of the individual discrepancies observed between lactate minimum and MLSS workloads $[4,12,13,23,26]$.

A limitation of the present investigation (studies 1 and 2) was that particular measurements, including pulmonary gas exchange, heart rate, and rating of perceived exertion, were not taken at the end of the lactate elevation phase in both lactate minimum tests (within each study), nor were they taken during the incremental phase. For coaches and daily clinical practice such measures are valuable as they provide additional indicators of training status/physiological exertion and can inform exercise training prescription.

In summary, although the $\left[\mathrm{lac}^{-}\right]_{\mathrm{B}}$ response to the incremental phase of our validated lactate minimum test protocol [11] is modified by a lower starting intensity and $1 \mathrm{~min}$ inter-stage rest intervals, the impact on lactate minimum power is minimal and unlikely to compromise test validity and therefore training status evaluation and exercise prescription. Our experience is that individuals of lower endurance training status with little familiarity of cycle exercise tolerate the validated protocol relatively poorly. Investigators may therefore wish to implement such protocol amendments for certain individuals/populations in order to make the test more tolerable. For daily practice and to inform future exercise prescription, concurrent measures of heart rate and rating of perceived exertion should also be taken. Furthermore, since $\mathrm{LM}_{40-60}$ and $\mathrm{LM}_{\mathrm{INT}}$ protocols decreased and increased, respectively, lactate minimum power by a similar amount ( 9 and $8 \mathrm{~W}$, respectively), combining the amendments may yield a lactate minimum power more similar to that produced by the previously validated protocol [11] and further improve participants' tolerance of the test. Future investigations should explore this possibility and evaluate the impact on MLSS prediction.

\section{References}

1 Bacon L, Kern M. Evaluating a test protocol for predicting maximum lactate steady state. J Sports Med Phys Fitness 1999; 39: 300-308

2 Bangsbo J, Graham T, Johansen L, Saltin B. Muscle lactate metabolism in recovery from intense exhaustive exercise: impact of light exercise. J Appl Physiol 1994; 77: 1890-1895

3 Beneke R, Hutler M, Von Duvillard SP, Sellens M, Leithauser RM. Effect of test interruptions on blood lactate during constant workload testing. Med Sci Sports Exerc 2003; 35: 1626-1630

4 Carter H, Jones AM, Doust JH. Effect of incremental test protocol on the lactate minimum speed. Med Sci Sports Exerc 1999; 31: 837-845

5 Denadai BS, Higino WP. Effect of the passive recovery period on the lactate minimum speed in sprinters and endurance runners. J Sci Med Sport 2004; 7: 488-496

6 Gladden LB. A lactatic perspective on metabolism. Med Sci Sports Exerc 2008; 40: 477-485

7 Gullstrand L, Sjodin B, Svedenhag J. Blood sampling during continuous running and 30-s intervals on a treadmill. Effects on the lactate threshold results? Scand J Med Sci Sports 1994; 4: 239-242

8 Harriss DJ, Atkinson G. International Journal of Sports Medicine - Ethical Standards in Sport and Exercise Science Research. Int J Sports Med 2009; 30: 701-702

9 Heck H, Mader A, Hess G, Mücke S, Müller R, Hollmann W. Justification of the 4-mmol/l lactate threshold. Int J Sports Med 1985; 6: 117-130

10 Hiyane WC, Simões HG, Campbell CSG. Critical velocity as a noninvasive method to estimate the lactate minimum velocity on cycling. Rev Bras Med Esporte 2006; 12: 340-344

11 Johnson MA, Sharpe GR, Brown PI. Investigations of the lactate minimum test. Int J Sports Med 2009; 30: 448-454

12 Jones AM, Doust JH. The validity of the lactate minimum test for determination of the maximal lactate steady state. Med Sci Sports Exerc 1998; 30: 1304-1313

13 MacIntosh BR, Esau S, Svedahl K. The lactate minimum test for cycling: estimation of the maximal lactate steady state. Can J Appl Physiol 2002; 27: 232-249 
14 Morris N, Gass G, Thompson M, Conforti D. Physiological responses to intermittent and continuous exercise at the same relative intensity in older men. Eur J Appl Physiol 2003; 90: 620-625

15 Ribeiro L, Balikian P, Malachias P, Baldissera V. Stage length, spline function and lactate minimum swimming speed. J Sports Med Phys Fitness 2003; 43: 312-318

16 Ribeiro LFP, Goncalves CGS, Kater DP, Lima MCS, Gobatto CA. Influence of recovery manipulation after hyperlactemia induction on the lactate minimum intensity. Eur J Appl Physiol 2009; 105: 159-165

17 Rotstein A, Dotan R, Zigel L, Greenberg T, Benyamini Y, Falk B. The effect of pre-test carbohydrate ingestion on the anaerobic threshold, as determined by the lactate-minimum test. Appl Physiol Nutr Metab 2007; 32: 1058-1064

18 Sabapathy S, Kingsley RA, Schneider DA, Adams L, Morris NR. Continuous and intermittent exercise responses in individuals with chronic obstructive pulmonary disease. Thorax 2004; 59: 1026-1031

19 Simões HG, Campbell CSG, Kokubun E, Denadai BS, Baldissera V. Blood glucose responses in humans mirror lactate responses for individual anaerobic threshold and for lactate minimum in track tests. Eur J Appl Physiol 1999; 80: 34-40

20 Simões HG, Denadai BS, Baldissera V, Campbell CSG, Hill DW. Relationships and significance of lactate minimum, critical velocity, heart rate deflection and $3000 \mathrm{~m}$ track-tests for running. J Sports Med Phys Fitness 2005; 45: 441-451
21 Simões HG, Hiyane WC, Sotero RC, Pardono E, Puga GM, Lima LC, Campbell CS. Polynomial modelling for the identification of lactate minimum velocity by different methods. J Sports Med Phys Fitness 2009; 49: $14-18$

22 Smith MF, Balmer J, Coleman DA, Bird SR, Davison RCR. Method of lactate elevation does not affect the determination of the lactate minimum. Med Sci Sports Exerc 2002; 34: 1744-1749

23 Sotero RC, Pardono E, Campbell CS, Simões HG. Indirect assessment of lactate minimum and maximal blood lactate steady-state intensity for physically active individuals. J Strength Cond Res 2009; 23: 847-853

24 Strupler M, Mueller G, Perret C. Heart rate-based lactate minimum test: a reproducible method. Br J Sports Med 2009; 43: 432-436

25 Svedahl K, MacIntosh BR. Anaerobic threshold: The concept and methods of measurement. Can J Appl Physiol 2003; 28: 299-323

26 Tegtbur U, Busse MW, Braumann KM. Estimation of an individual equilibrium between lactate production and catabolism during exercise. Med Sci Sports Exerc 1993; 25: 620-627

27 Turner PT, Cathcart AJ, Parker ME, Butterworth C, Wilson J, Ward SA. Oxygen uptake and muscle desaturation kinetics during intermittent cycling. Med Sci Sports Exerc 2006; 38: 492-503 